\title{
Neurociencia afectiva del TDAH: Datos existentes y direcciones futuras
}

\section{Affective neuroscience of ADHD: Current data and future directions}

\author{
Sara López-Martín ${ }^{1}$, Jacobo Albert ${ }^{1}$, Alberto Fernández-Jaén ${ }^{2}$, Luis Carretié ${ }^{1}$ \\ ${ }^{1}$ Departamento de Psicología Biológica y de la Salud. Facultad de Psicología. Universidad Autónoma de Madrid. Madrid, España.
} ${ }^{2}$ Unidad de Neurología Infantil. Hospital Quirón. Madrid, España.

Disponible online 30 de abril de 2010

\begin{abstract}
El trastorno por déficit de atención con hiperactividad (TDAH) es un trastorno de origen neurobiológico complejo y heterogéneo que se caracteriza no sólo por sus disfunciones cognitivas sino también por la existencia de importantes alteraciones afectivas, tanto motivacionales como emocionales. Sin embargo, los correlatos neurobiológicos que subyacen a las disfunciones afectivas del TDAH apenas han sido explorados, en comparación con la extensa investigación llevada a cabo sobre los mecanismos neurales implicados en sus principales déficit cognitivos (atención, inhibición de respuesta y memoria de trabajo). Este trabajo revisa las recientes investigaciones que han explorado las bases neurales involucradas en las alteraciones motivacionales y emocionales mostradas por las personas con TDAH. Asimismo, se discuten las implicaciones prácticas derivadas de los resultados de estos estudios y se proponen nuevas líneas de investigación desde la Neurociencia afectiva.
\end{abstract}

Palabras clave: Trastorno por déficit de atención con hiperactividad; TDAH; Emoción; Motivación; Recompensa.

Attention-deficit/hyperactivity disorder (ADHD) is a complex and heterogeneous disorder that is characterized not only by cognitive deficits but also by affective dysfunctions, both motivational and emotional. Nevertheless, the neural bases of affective dysfunctions have barely been explored in relation to this disorder, in contrast to extensive research that examined the neural correlates of its main cognitive deficits (attention, response inhibition and working memory). This article reviews the available data regarding the neurobiological substrates of motivational and emotional alterations showed by children, adolescents and adults with ADHD. Practical implications derived from these data are discussed and future research directions from affective neuroscience are suggested.

Keywords: Attention-deficit/hyperactivity disorder; ADHD; Emotion; Motivation; Reward.

Estudio financiado por el Ministerio de Ciencia e Innovación (PSI2008-03688) y la Comunidad de Madrid/Universidad Autónoma de Madrid (CCG08-UAM/SAL-4463).

Correspondencia: Sara López Martín. Departamento de Psicología Biológica y de la Salud. Facultad de Psicología. Universidad Autónoma de Madrid. C/ Ivan Pavlov, No 6. 28049, Madrid. E-mail: sara.lopez@uam.es 
El trastorno por déficit de atención con hiperactividad (TDAH) es la etiqueta diagnóstica empleada actualmente para denominar a uno de los trastornos del neurodesarrollo más frecuentes en población infantil y, sin duda, uno de los que mayor polémica suscita. El empleo de metilfenidato, un psicoestimulante del sistema nervioso central, como uno de los principales tratamientos de un trastorno diagnosticado a partir de la conducta del paciente, genera desconfianza en las familias y en la sociedad. Por otro lado, el creciente número de niños diagnosticados con TDAH en los últimos años ha generado un debate público sobre las causas que lo originan, la validez de su diagnóstico y los efectos adversos del tratamiento farmacológico. Sin embargo, la evidencia científica que apoya la heredabilidad del trastorno, así como la existencia de anomalías cerebrales anatómicas y funcionales es extensa y robusta. En efecto, el TDAH tiene un fuerte componente genético, como así lo indican los estudios de niños adoptados y los estudios de gemelos (Albert, López-Martín, Fernández-Jaén y Carretié, 2009). Los primeros han observado que la prevalencia del trastorno es claramente mayor entre los familiares biológicos que entre los familiares adoptivos (Sprich, Biederman, Crawford, Mundy y Faraone, 2000). Los segundos estiman que el índice heredabilidad del trastorno es de 0,77 (Faraone et al., 2005), una cifra elevada si se compara con la de otros trastornos neuropsquiátricos como la depresión $(0,39)$ o la ansiedad generalizada $(0,32)$. Por otro lado, datos convergentes de estudios neuropsicológicos, neuroquímicos y de neuroimagen estructural y funcional han mostrado que el TDAH está caracterizado por anomalías en una amplia variedad de regiones cerebrales, entre las que destacan aquellas que forman el circuito fronto-estriado: corteza prefrontal dorsolateral y regiones dorsales de la corteza cingulada anterior y el estriado (Bush, Valera y Seidman, 2005; Seidman, Valera y Makris, 2005; Willcutt, Doyle, Nigg, Faraone y Pennington, 2005; Zimmer, 2009). La disfunción de este circuito parece ser la responsable de los déficit ejecutivos, y especialmente del pobre control inhibitorio, mostrados por muchos niños, adolescentes y adultos con TDAH.

El desarrollo en la última década de modernas técnicas de estudio del cerebro, tanto a nivel anatómico como funcional, ha permitido conocer las importantes diferencias entre el cerebro con TDAH y el cerebro sano. Por un lado, un amplio número de estudios que han empleado diversas técnicas anatómicas muestran que, como grupo, el volumen cerebral de los niños con TDAH es menor que el de los niños control, tanto si se compara el volumen de todo el cerebro (la reducción es de aproximadamente un 3-5\%: Seidman et al., 2005) como si se tienen en cuenta determinadas regiones cerebrales (regiones de la corteza prefrontal, caudado, vermis y esplenio del cuerpo calloso: Valera, Faraone, Murray y Seidman, 2007). Por otro lado, los estudios que han empleado técnicas funcionales, tanto hemodinámicas como electrofisiológicas, han observado de forma consistente una disfunción del circuito fronto-estriado mientras las personas con TDAH realizaban tareas cognitivas sin contenido afectivo que involucraban, fundamentalmente, a la atención y al control inhibitorio (Barry, Johnstone y Clarke, 2003; Bush et al., 2005; Dickstein, Bannon, Castellanos y Milham, 2006). Entre las tareas que más se han empleado para demostrar la disfunción de este circuito se encuentran aquellas que requieren la inhibición de una repuesta prepotente (Go/Nogo), la inhibición de una respuesta ya iniciada (Stop-signal) y la inhibición de información contradictoria o irrelevante (Flanker). No obstante, aunque la evidencia científica que apoya la disfunción del circuito fronto-estriado en el TDAH es extensa y robusta, cada vez es más evidente que las alteraciones de este circuito no son suficientes para explicar la enorme heterogeneidad del trastorno. Otras regiones y circuitos neurales, como el cerebelo o los sistemas mesolímbico y parieto-temporal (Vaidya y Stollstorff, 2008), están comprometidos en el TDAH.

La heterogeneidad del TDAH no sólo se pone de manifiesto a nivel neurobiológico, con la implicación de distintos circuitos neurales, sino también en otros niveles de análisis (Figura 1): desde las causas que lo originan (genéticas, ambientales) y la implicación de múltiples genes (ADRA2A, SLC6A2, DRD4, DRD5, DAT) y neurotransmisores (dopamina, noradrenalina, serotonina), hasta las diferencias en la expresión de los síntomas (inatención, hiperactividad, impulsividad) y la comorbilidad con otros trastornos neuropsiquiátricos (trastornos de conducta, trastornos de aprendizaje, ansiedad, depresión). A nivel neuropsicológico, esta heterogeneidad se refleja en la alteración de una amplia variedad de procesos psicológicos, tanto cognitivos (atención, inhibición de respuesta, memoria de trabajo, procesamiento temporal) como afectivos (procesamiento de la recompensa, aversión a la demora, procesamiento emocional, regulación emocional). Sin embargo, mientras que las deficiencias cognitivas, y especialmente las ejecutivas, han sido ampliamente estudiadas, apenas se dispone de información sobre las disfunciones afectivas del TDAH. En efecto, durante muchos años se ha visto el TDAH como un trastorno cognitivo en el que sus principales síntomas estaban originados por una disfunción ejecutiva cuyo principal proceso implicado era la inhibición de respuesta (Barkley, 1997). No obstante, recientemente se han propuesto diversas teorías multicausales que tienen en cuenta la heterogeneidad del trastorno y que incorporan no sólo procesos cognitivos sino también afectivos (Nigg y Casey, 2005; Sonuga-Barke, 2002). El desarrollo de estos nuevos modelos ha fomentado el estudio de las alteraciones afectivas que caracterizan a muchas de las personas con TDAH, así como la exploración de las bases neurobiológicas que las sustentan. Asimismo, el interés de los investigadores por el estudio de las alteraciones emocionales y motivacionales del TDAH se ha incrementado en los últimos años al conocer los resultados de varios estudios que indican que los déficit en las funciones ejecutivas no son necesarios ni suficientes para explicar todos los casos de TDAH (Willcutt et al., 2005). Otros procesos psicológicos, tanto cognitivos como afectivos, tienen necesariamente que estar detrás de este trastorno. 
Figura 1. Heterogeneidad del TDAH en todos los niveles de análisis: desde las causas que lo producen hasta la aparición de distintos trastornos asociados. La Figura no es exhaustiva. Otros circuitos cerebrales, procesos psicológicos o trastornos asociados pueden estar involucrados en el TDAH.

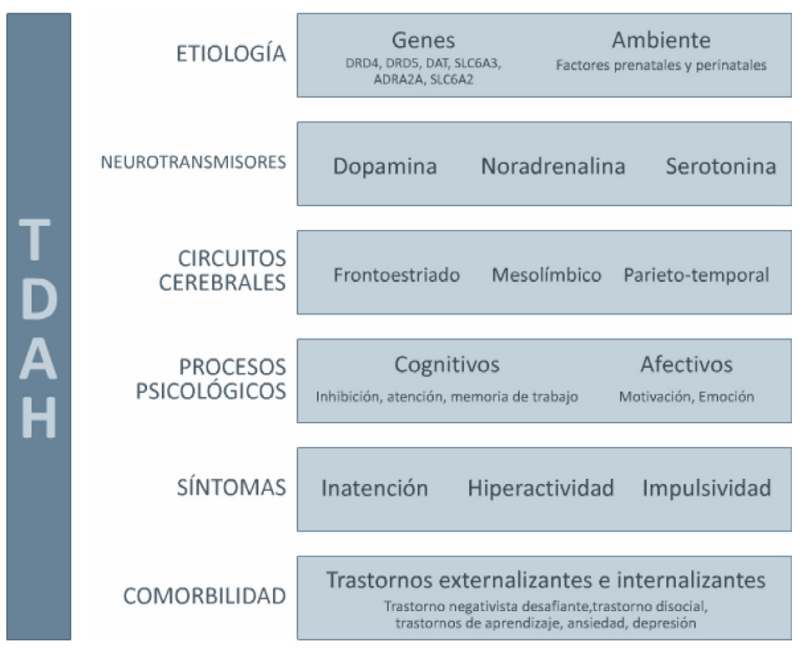

El presente artículo pretende revisar la investigación realizada hasta el momento sobre las alteraciones neurobiológicas que subyacen a las disfunciones motivacionales y emocionales que presentan muchos de los niños, adolescentes y adultos con TDAH. Dado el carácter incipiente de la investigación sobre este asunto, los resultados que aquí se exponen deben interpretarse con cautela, en espera de que sean replicados por futuras investigaciones. Asimismo, se discuten las implicaciones clínicas de estos nuevos hallazgos y se proponen nuevas líneas de investigación sobre el TDAH desde la Neurociencia afectiva.

Alteraciones motivacionales en el TDAH

La idea de que el TDAH está asociado con anomalías en el sistema de recompensa tiene una larga historia (Douglas y Parry, 1983, 1994; Rapport, Tucker, DuPaul, Merlo y Stoner, 1986; Sagvolden, Aase, Zeiner y Berger, 1998; Sonuga-Barke, 2002; Sonuga-Barke, Dalen y Remington, 2003; SonugaBarke, Taylor, Sembi y Smith, 1992;Tripp y Alsop, 2001). No obstante, el estudio de las alteraciones motivacionales siempre ha estado en un segundo plano debido a que el foco de interés de clínicos e investigadores se ha centrado en la búsqueda y en la

Figura 2. En este diagrama se observa cómo, de forma general, las recompensas son más eficaces cuando se reciben de forma inmediata a la realización de la conducta/respuesta (línea gris oscuro). De esta forma, a medida que aumenta el intervalo temporal entre la conducta/ respuesta $(\mathrm{Ra}, \mathrm{Rb}, \mathrm{Rc}, . ., \mathrm{Rn})$ y el momento en que se recibe la recompensa por la misma, se produce un descenso en el valor/eficacia de dicha recompensa (por lo que ésta tendrá una menor influencia sobre el comportamiento). Por otra parte, se observa también que en el caso de las personas con TDAH (línea gris claro), este descenso en el valor de la recompensa es más acusado que en el caso de los controles (la pendiente es más acentuada), de forma que intervalos temporales más pequeños se asocian a una pérdida más rápida del valor de la recompensa. Figura adaptada de Sagvolden, Johansen, Aase y Russell (2005). comprensión de las disfunciones ejecutivas del TDAH. Este es el motivo por el que los datos existentes sobre las alteraciones motivacionales en el TDAH no sean ni abundantes ni en muchos casos coincidentes. Sin embargo, la revisión de Luman, Oosterlann y Sergeant (2005) sobre este asunto permite llegar a las siguientes conclusiones: a) las personas con TDAH dependen en mayor medida que los controles del refuerzo externo para obtener su máximo rendimiento, b) las personas con TDAH normalizan su rendimiento cuando se les refuerza frecuentemente y de forma inmediata, c) las personas con TDAH muestran una menor respuesta psicofisiológica periférica (tasa cardiaca y conductancia de la piel) ante la recompensa que las personas sin TDAH, y d) en situaciones en las que se debe elegir entre una recompensa inmediata y otra demorada, las personas con TDAH escogen más a menudo que los controles las recompensas inmediatas, incluso cuando las recompensas demoradas son mayores/mejores que las recompensas inmediatas. Esta última característica, es decir, la preferencia por las recompensas inmediatas frente a las demoradas, es el hallazgo más consistente de la investigación sobre las alteraciones motivacionales del TDAH (Antrop, Verte y Baeyens, 2006; Rapport, et al., 1986; Sonuga-Barke et al., 1992). Además, constituye una disfunción primaria del TDAH y, por tanto, independiente de las disfunciones ejecutivas características del trastorno (Antrop et al., 2006; Solanto et al., 2001; Sonuga-Barke et al., 2003; Thorell, 2007).

Diversos modelos motivacionales se han propuesto en los últimos años como alternativa a las tradicionales teorías cognitivas del TDAH (véase una excelente revisión sobre este asunto en Luman, Tripp y Scheres, 2010). Posiblemente los más influyentes son la teoría del desarrollo dinámico (Sagvolden, Johansen, Aase y Russell, 2005), la teoría del déficit en la transferencia de dopamina (Tripp y Wickens, 2008) y la hipótesis de la aversión a la demora (Sonuga-Barke, 2002, 2003). Los tres modelos sostienen que las alteraciones motivacionales del TDAH están originadas por una baja activación del sistema de recompensa dopaminérgico, aunque los dos primeros lo hacen desde un punto de vista neuroquímico y el tercero desde un punto de vista anatómico-funcional. De forma resumida, la teoría del desarrollo dinámico de Sagvolden et al. (2005) sostiene que un bajo nivel general de dopamina en el sistema mesolímbico es el responsable de la presencia en el TDAH de un gradiente de demora de la recompensa más corto y pronunciado (Figura 2).

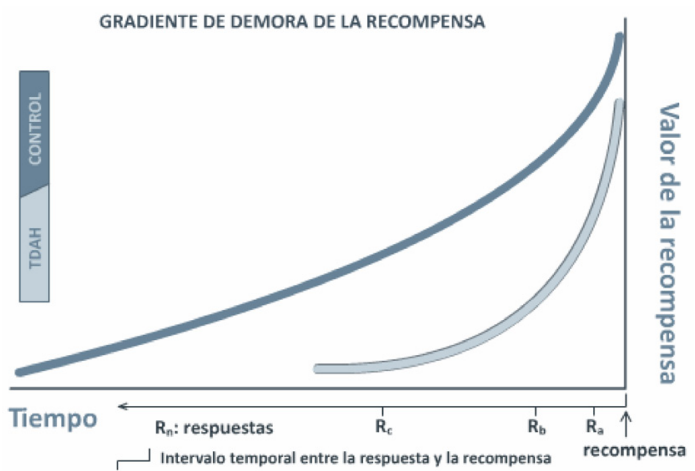


Por su parte, la teoría del déficit en la transferencia de dopamina de Tripp y Wickens (2008) propone que algunos de los síntomas del TDAH podrían explicarse por una disminución específica de la tasa de disparo de la célula dopaminérgica en la anticipación de la recompensa. Estos autores argumentan que en las personas sanas, la respuesta de la célula dopaminérgica ante un refuerzo positivo se transfiere a señales previas que predicen la posterior recompensa. Esta transferencia proporciona refuerzo inmediato a nivel celular cuando la entrega de la recompensa se demora. Sin embargo, en las personas con TDAH la transferencia de la respuesta de la célula dopaminérgica a la señal que predice la posterior entrega de la recompensa no se produce correctamente (Figura 3).

Figura 3. Teoría del déficit en la transferencia de dopamina (Tripp y Wickens, 2008). En las personas sin TDAH (línea gris oscuro) se produce una transferencia de la respuesta de la célula dopaminérgica, produciéndose primero ante la recompensa para posteriormente (mediante el aprendizaje) producirse ante la señal que la predice. En las personas con TDAH (línea gris claro) esta transferencia no se produciría correctamente. Figura adaptada de Tripp y Wickens (2008).
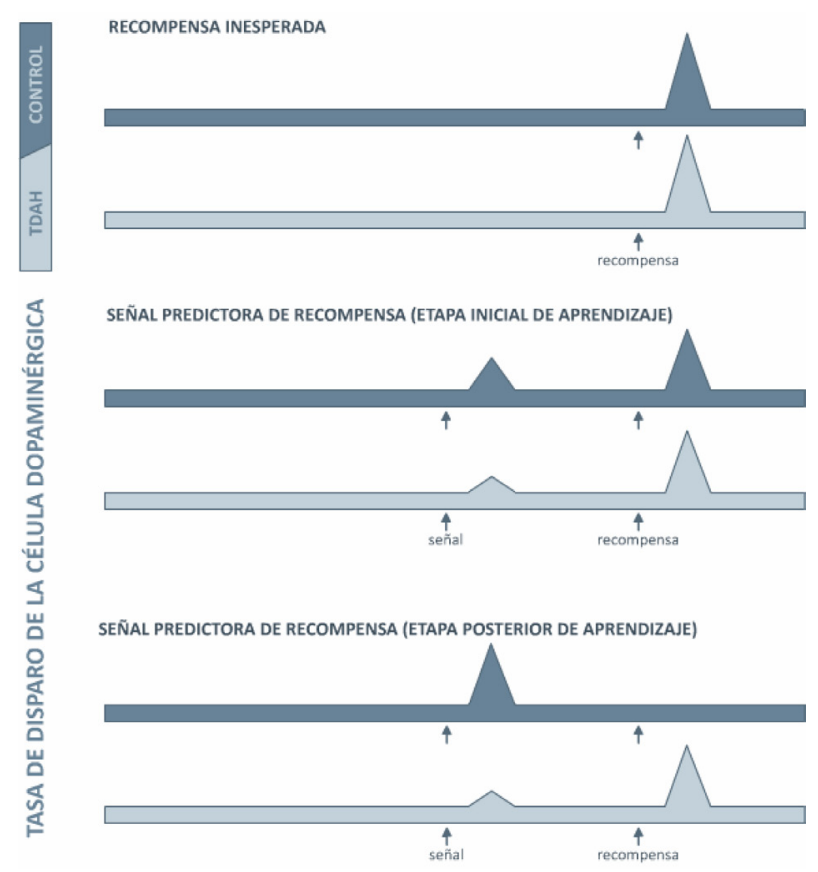

Finalmente, el modelo de doble vía de Sonuga-Barke (2002, 2003), en el que está actualmente incluida la hipótesis de la aversión a la demora, propone que en el TDAH están alterados, al menos, dos sistemas neurales que además son independientes: el circuito fronto-estriado y el circuito mesolímbico. El primero de ellos estaría detrás de las disfunciones ejecutivas, mientras que el segundo explicaría los problemas motivacionales. Sonuga-Barke propone que la aversión a la demora característica del TDAH se refleja a nivel conductual en la preferencia de recompensas pequeñas e inmediatas frente a recompensas mayores pero demoradas. De acuerdo con este modelo, escapar a la demora constituye el principal reforzador (en este caso, negativo) de las personas con TDAH, ya que éstas perciben la demora como algo negativo o desagradable. Este modelo predice además que en las situaciones en las que no sea posible reducir la espera hasta conseguir la recompensa demorada (por ejemplo, al no existir refuerzos inmediatos), las personas con TDAH intentarán reducir la experiencia subjetiva de espera dirigiendo su atención a otros estímulos e incrementando el nivel de estimulación a través del movimiento.

En general, los datos conductuales disponibles hasta el momento y las predicciones realizadas por los principales modelos motivacionales del TDAH encajan bien con los recientes hallazgos obtenidos sobre las bases neurobiológicas que subyacen al procesamiento de la recompensa en adolescentes y adultos con el trastorno. En efecto, a pesar de que los estudios que han explorado los sustratos neurales de las alteraciones motivacionales en el TDAH son escasos, los resultados obtenidos hasta el momento son coincidentes y a su vez complementarios, ya que indican que estas alteraciones están originadas por anomalías anatómicas (Carmona et al., 2009), neuroquímicas (Volkow et al., 2007, 2009) y funcionales (tanto electrofisiológicas como hemodinámicas: Holroyd, Baker, Kerns y Müller, 2008; Plichta et al., 2009; Scheres, Milham, Knutson y Castellanos, 2007; Ströhle et al., 2008) en las regiones cerebrales que forman el sistema de recompensa: fundamentalmente, la corteza ventromedial (aquí definida como un área extensa que incluye a las cortezas prefrontal medial y orbital: Carretié, López-Martín y Albert, 2010) y la región ventral del estriado, cuya principal estructura es el núcleo accumbens.

En primer lugar, el estudio llevado a cabo recientemente por Carmona et al. (2009) sugiere por primera vez la presencia de anomalías estructurales en regiones cerebrales asociadas con la motivación y el procesamiento de la recompensa. En efecto, estos investigadores han observado que los niños con TDAH presentan reducciones significativas en los volúmenes de las regiones ventrales del estriado. Asimismo, observaron que el volumen de la región ventral derecha del estriado correlacionaba negativamente con los síntomas de hiperactividad e impulsividad pero no con los síntomas de inatención. Estos resultados concuerdan con los estudios realizados con animales que muestran respuestas impulsivas en ratas tras lesiones del núcleo accumbens, lo que sugiere que esta región podría estar involucrada en la patogénesis de la impulsividad y la hiperactividad (Cardinal, Pennicott, Sugathapala, Robbins y Everitt, 2001).

En segundo lugar, el grupo de Volkow (2007, 2009) encontró recientemente anomalías neuroquímicas en regiones cerebrales claves en el sistema de recompensa en adultos con TDAH. En concreto, estos investigadores observaron mediante el empleo de la tomografía por emisión de positrones (TEP) una menor disponibilidad de algunos receptores (D2/D3) y transmisores (DAT) dopaminérgicos en el núcleo accumbens, 
el mesencéfalo y el hipotálamo. En todas las regiones se observaron estos cambios en el hemisferio izquierdo. Además, hallaron una relación inversa entre la disponibilidad de receptores D2/D3 en el núcleo accumbens y los síntomas de inatención, lo que sugeriría que las alteraciones en el sistema de recompensa también podrían estar vinculadas con los problemas atencionales característicos del TDAH. Este hecho podría explicar por qué las personas con TDAH tienen mayores dificultades para mantener la atención en tareas aburridas y repetitivas (es decir, con una baja capacidad para reforzar). Además, la menor disponibilidad de los receptores de dopamina D2/D3 en el núcleo accumbens podría explicar la mayor vulnerabilidad de los adolescentes y adultos con TDAH al consumo de drogas, ya que se ha constatado que las alteraciones en los receptores de dopamina D2/D3 están asociadas con un mayor riesgo de abuso de sustancias, tanto en ratas (Dalley et al., 2007) como en humanos (Lee et al., 2009). Finalmente, la menor disponibilidad de receptores D2/D3 en el hipotálamo en los adultos con TDAH proporciona una posible base neurobiológica para explicar por qué las personas con TDAH presentan con asiduidad problemas de sueño (Sung, Hiscock, Sciberras y Efron, 2008) y sobrepeso (Cortese et al., 2008), así como una respuesta anómala ante el estrés (van West, Claes y Deboutte, 2009). En todo caso, los importantes hallazgos del grupo de Volkow et al. (2009) deben interpretarse con cautela, ya que no es posible establecer una relación directa entre la disponibilidad de marcadores sinápticos de dopamina en el mesoaccumbens y la respuesta anormal a la recompensa de los adultos con TDAH. Es decir, esta investigación no empleó tarea experimental alguna para evaluar directamente la respuesta neuroquímica de los participantes cuando eran recompensados.

Los estudios de Scheres (2007) y Ströhle (2008) con resonancia magnética funcional dan un paso más al observar un funcionamiento anómalo del sistema de recompensa en adolescentes y adultos con TDAH, respectivamente. En ambas investigaciones se encontró en las personas con TDAH una activación reducida del estriado ventral durante la anticipación de la recompensa. En otras palabras, los participantes con TDAH no lograron activar tan intensamente como los controles las regiones ventrales del estriado cuando una señal les anticipaba que posteriormente iban a ganar dinero. Sin embargo, ninguno de los dos estudios observó diferencias en la actividad cerebral entre ambos grupos cuando la señal anticipaba una pérdida económica en lugar de una ganancia. En este sentido, estos datos sugieren que las alteraciones motivacionales en el TDAH se asocian fundamentalmente con el procesamiento de la recompensa, sin implicar a otros procesos motivacionales como el castigo. Por otro lado, ambos estudios observaron que la activación del estriado ventral correlacionaba con la hiperactividad y la impulsividad: una menor activación de esta región durante la espera de la recompensa se asociaba con mayores niveles de hiperactividad e impulsividad. Además de la hipoactivación del estriado ventral durante la anticipación de la recompensa, Ströhle et al. (2008) observaron que en los adultos con TDAH se producía un incremento de la actividad de la corteza orbitofrontal cuando se les informaba sobre la recompensa económica que habían obtenido. Aunque este hallazgo todavía no tiene una interpretación clara, datos obtenidos en población no clínica indican que la corteza orbitofrontal juega un papel importante en el aprendizaje y en el valor otorgado a una recompensa (Rolls, 2000).

Recientemente, Plichta et al. (2009) compararon la activación cerebral de adultos sanos y adultos con TDAH mientras realizaban una tarea experimental que requería escoger entre dos recompensas económicas que variaban en la demora en las que eran entregadas. Por un lado, los adultos con TDAH mostraron una menor activación de la región ventral del estriado en comparación con los adultos sanos tanto para las recompensas más inmediatas como para las más demoradas. Este hallazgo está en consonancia con los datos obtenidos por los estudios previos de actividad cerebral (Scheres et al., 2007; Ströhle et al., 2008) así como por los estudios que han hallado una reducida respuesta psicofisiológica (tasa cardiaca y conductancia de la piel) ante la recompensa en personas con TDAH (Luman et al., 2005; Luman, Oosterlaan, Hyde, van Meel y Sergeant, 2007). Además, Plichta et al. (2009) observaron que los adultos con TDAH mostraban una mayor activación de la región dorsal del estriado y de la amígdala cuando escogían la recompensa demorada en lugar de la recompensa más inmediata. Estos datos sugieren que las personas con TDAH experimentan la elección de la recompensa demorada como una pérdida al dejar pasar la oportunidad de obtener el dinero de forma inmediata. En este sentido, los datos obtenidos dentro de la investigación sobre drogas en personas adictas indican que un incremento en la activación del caudado se asocia con mayores niveles de deseo y ansias por conseguir la droga. Por otro lado, la hiperactivación de la amígdala ante las recompensas demoradas sugiere que las personas con TDAH experimentan la espera como algo negativo y desagradable, lo que apoyaría las predicciones de la hipótesis de la aversión a la demora (Sonuga-Barke, 2002; 2003).

\section{Alteraciones emocionales en el TDAH}

Reconocer correctamente las emociones que están experimentando otros y regular de forma efectiva nuestras propias emociones son importantes habilidades que facilitan las interacciones sociales. Los estados emocionales y las intenciones se hacen visibles a través de señales afectivas como las expresiones faciales y la prosodia. Por tanto, la habilidad para interpretar y responder apropiadamente a estas señales constituye un componente fundamental para desarrollar relaciones interpersonales adecuadas. De hecho, las dificultades en el reconocimiento emocional están relacionadas con las importantes deficiencias sociales que presentan tanto las personas con trastornos del espectro autista (p.e., Critchley et al., 2000) como 
las personas con esquizofrenia (Marsh y Williams, 2006). Por otro lado, la habilidad para regular las emociones es crucial no sólo para un buen funcionamiento social sino también para el bienestar general. En este sentido, un pobre control emocional parece estar en la base de la depresión y la ansiedad (Gross, 1998), así como de los trastornos asociados con el abuso de sustancias (Goldstein y Volkow, 2002).

A continuación se describen las principales investigaciones que han abordado el estudio de la competencia emocional en niños, adolescentes y adultos con TDAH. Los datos existentes se organizan en dos grandes grupos: los relacionados con el procesamiento emocional y los asociados con la expresión y la regulación emocional. La investigación sobre las alteraciones emocionales del TDAH carece, a diferencia del estudio de las disfunciones motivacionales, de un marco teórico bien definido. La teoría integradora de Nigg y Casey (2005), es posiblemente la más explícita en este sentido, ya que sostiene que el TDAH es el resultado de un mal funcionamiento de tres circuitos neurales entre los que se incluye uno que está implicado en la evaluación del significado emocional de los acontecimientos (circuito prefrontal-amigdalar). Asimismo, algunas de las principales teorías motivacionales del TDAH, como el modelo de doble vía (Sonuga-Barke, 2002, 2003) pueden ser útiles para interpretar algunos de los resultados que se exponen a continuación. De hecho, la hipótesis de la aversión de la demora de Sonuga-Barke $(2002,2003)$ predice una hiperactivación de estructuras asociadas con la evaluación emocional (como por ejemplo, la amígdala) cuando las personas con TDAH tienen que esperar la recompensa, ya que perciben la demora como algo negativo o desagradable.

\section{Reconocimiento emocional en el TDAH}

Datos convergentes procedentes de estudios conductuales indican que tanto los niños como los adultos con TDAH muestran importantes dificultades para reconocer y comprender la información afectiva (Albert, López-Martín, Fernández-Jaén y Carretié, 2008). En este sentido, diversos investigadores han observado que los niños y adultos con TDAH obtienen peores resultados que los controles en múltiples tareas de reconocimiento emocional que requieren la identificación de emociones básicas a partir de la expresión facial y la prosodia (Cadesky, Mota y Schachar, 2000; Corbett y Glidden, 2000; Norvilitis, Casey, Brooklier y Bonello, 2000; Shapiro, Hughes, August y Bloomquist, 1993; Singh et al., 1998). Un estudio reciente ha mostrado además que los niños y adolescentes con TDAH son menos precisos que los controles no sólo cuando tienen que reconocer una emoción a partir de su expresión facial sino también cuando tienen que utilizar la información contextual para comprender la emoción que está experimentando una persona (Da Fonseca, Seguier, Santos, Poinso y Deruelle, 2009). Por tanto, estos datos sugieren la presencia de un déficit general en el procesamiento emocional en el TDAH, el cual además parece estar relacionado con los problemas interpersonales y las bajas habilidades sociales observadas en muchos niños y adultos con el trastorno (Kats-Gold, Besser y Priel, 2007).

Una vez demostrada la existencia de un déficit en el procesamiento emocional en el TDAH, el siguiente paso debe consistir en buscar las causas que lo producen. Por un lado, no cabe duda de que algunos de los síntomas más característicos del TDAH, como la falta de atención o la impulsividad, pueden dificultar el reconocimiento de señales afectivas que además son a menudo sutiles (p.e., una mirada o un gesto). En este caso, el déficit en el procesamiento emocional no constituiría una disfunción per se del TDAH sino un problema secundario originado por uno o varios de sus déficit cognitivos. En este sentido, los datos de algunos estudios sugieren que efectivamente determinadas deficiencias cognitivas características del TDAH, especialmente la falta de atención, dificultan el reconocimiento de las expresiones faciales emocionales (Shapiro et al., 1993). Sin embargo, datos recientes procedentes de distintas investigaciones señalan que las dificultades en el reconocimiento emocional no pueden explicarse únicamente por las disfunciones cognitivas características del trastorno (Rapport et al., 2002; Yuill y Lyon, 2007; Da Fonseca et al., 2009). Por tanto, según estos últimos datos, las dificultades en el reconocimiento emocional constituirían una disfunción primaria del TDAH. En todas estas investigaciones, los participantes realizaron tareas de control emocionalmente neutras que requerían la activación de las mismas habilidades cognitivas que son necesarias para completar las tareas de reconocimiento emocional, observándose que las personas con TDAH tenían un rendimiento similar al grupo control en las tareas control sin contenido afectivo, mientras que diferían significativamente de sus iguales en las tareas de reconocimiento emocional. Estas dificultades se han observado en niños y en adultos con TDAH cuando tenían que identificar distintas emociones en caras humanas (Rapport et al., 2002; Yuill y Lyon, 2007) y también cuando debían inferir las emociones que experimentaba una persona a través de la información contextual (Da Fonseca et al., 2009). Por tanto, estos resultados indican que los problemas en el reconocimiento emocional mostrados por niños, adolescentes y adultos con TDAH no pueden explicarse únicamente por la impulsividad, la falta de atención o cualquier otra deficiencia cognitiva o perceptiva.

El próximo paso debe consistir en examinar las bases neurobiológicas que subyacen a los problemas en el procesamiento emocional mostrados por niños y adultos con TDAH. Williams et al. (2008) fueron los primeros en utilizar tanto medidas conductuales como medidas de actividad cerebral para evaluar el procesamiento emocional en personas con TDAH. A nivel conductual, observaron que los niños y adolescentes con TDAH cometían más errores que los controles cuando tenían que identificar emociones negativas, como el miedo o el enfado, en caras humanas. A nivel neural, la anomalía más relevante con- 
sistió en una reducción, en regiones occipitales, del componente P120 de los potenciales evento-relacionados (PER) o potenciales evocados en los pacientes con TDAH en comparación con las personas sanas, también ante las caras de enfado y miedo. El componente P120 está asociado con un análisis temprano y posiblemente automático de la percepción de la emoción en caras humanas (Williams, Palmer, Liddell, Song y Gordon, 2006). Por otro lado, estos investigadores sugieren que estas alteraciones tempranas en el procesamiento emocional de los niños y adolescentes con TDAH podrían estar relacionadas con una disfunción de la amígdala, ya que ésta modula la actividad de las áreas sensoriales a través de sus proyecciones a las cortezas sensoriales, tanto visual como auditiva. La posible implicación de la amígdala podría explicar las correlaciones observadas por estos autores entre la amplitud de este componente y los niveles de ansiedad, depresión y labilidad emocional mostrados por los pacientes con TDAH. Finalmente, el grupo de Williams (2008) también observó que el tratamiento farmacológico con metilfenidato normalizaba la amplitud del componente P120 de los niños y adolescentes con TDAH. Por tanto, estos resultados sugieren que el metilfenidato no sólo normaliza el funcionamiento de diversos procesos cognitivos, como la atención y el control inhibitorio, sino también de otros procesos afectivos como, en este caso, el procesamiento emocional.

Por otro lado, Herrmann et al. (2009) exploraron recientemente el procesamiento emocional de los adultos con TDAH utilizando imágenes emocionales en lugar de expresiones faciales emocionales. Estos autores observaron también anomalías electrofisiológicas asociadas con el procesamiento emocional en áreas occipitales del cuero cabelludo. Sin embargo, en este caso encontraron una reducción de la respuesta cerebral ante la estimulación emocionalmente positiva. En concreto, estos investigadores observaron una menor amplitud de un componente de los PER denominado Negatividad temprana posterior (NTP) en los adultos con TDAH en comparación con los adultos sanos ante las imágenes positivas, no encontrándose diferencias entre ambos grupos ante las imágenes negativas. La NTP refleja una facilitación del procesamiento de los estímulos emocionales, tanto positivos como negativos, posiblemente debida a una mayor activación de los mecanismos neurales asociados con la atención selectiva (Schupp, Junghöfer, Weike y Hamm, 2003). Estos hallazgos coinciden con los datos preliminares obtenidos por López-Martín et al. (López-Martín, Albert, Fernández-Jaén y Carretié, 2008) con niños con TDAH utilizando igualmente estímulos visuales emocionales. Estos últimos también observaron una reducida activación de los mecanismos neurales asociados con la atención selectiva en los niños con TDAH en comparación con los niños sanos ante estímulos visuales positivos (no observándose diferencias ante las imágenes negativas y neutras). Por tanto, ambos estudios sugieren que las personas con TDAH mostrarían una respuesta reducida no sólo ante las recompensas sino ante todo tipo de estímulos agradables o positivos.
Nuevos estudios deberán explorar si las deficiencias en el procesamiento de la recompensa y en el procesamiento emocional constituyen disfunciones independientes o si, por el contrario, son expresiones de un mismo déficit afectivo. Por otro lado, es necesario el empleo de técnicas hemodinámicas para poder examinar el funcionamiento de algunas regiones subcorticales, como la amígdala y el estriado, ya que ambas juegan un papel importante en el procesamiento de la información afectiva (Carretié et al., 2009a, 2009b). De hecho, el reciente estudio de Brotman et al. (2010) con resonancia magnética funcional ha mostrado por primera vez de manera directa la existencia de una disfunción de la amígdala durante una tarea de procesamiento emocional en pacientes con TDAH.

\section{Expresión y regulación emocional en el TDAH}

Los estudios que han examinado la regulación emocional en niños y adolescentes con TDAH sugieren que tanto unos como otros tienen importantes dificultades para controlar sus emociones, especialmente cuando éstas son negativas (Albert et al., 2008). Los datos obtenidos a partir de distintos estudios conductuales indican que los niños y adolescentes con TDAH expresan mayores niveles de depresión (Kitchens, Rosén y Braaten, 1999; Williams et al., 2008), agresividad (Kitchens et al., 1999; Maedgen y Carlson, 2000), tristeza (Braaten y Rosén, 2000) y enfado (Braaten y Rosén, 2000; Ramírez et al., 1997; Richards, Deffenbacher, Rosen, Barkley y Rodricks, 2006) que sus iguales sin TDAH. Esta excesiva reactividad emocional mostrada por los niños y adolescentes con TDAH se ha observado mientras realizaban deportes individuales y colectivos (Clendenin, Businelle y Kelley, 2005; Johnson y Rosen, 2000) y en adultos durante la conducción de sus vehículos (Richards et al., 2006). Recientes investigaciones muestran además que los niños con TDAH son incapaces de ocultar sus emociones incluso después de recibir instrucciones para hacerlo (Walcott y Landau, 2004). Igualmente, se ha observado que tanto los adolescentes como los adultos con TDAH muestran una excesiva labilidad emocional (p.e., Sobanski et al., en prensa). En este sentido, los estudios realizados por el grupo de Wender y Reimherr (Reimherr et al., 2005; Wender, Reimherr y Wood, 1981; Wender, Reimherr, Wood y Ward, 1985) ponen de manifiesto que los adultos con TDAH no sólo presentan dificultades cognitivas (p.e., desorganización, impulsividad e inatención), sino también distintos problemas afectivos relacionados con una pobre regulación emocional: labilidad emocional pronunciada, excesiva reactividad emocional y un carácter fácilmente irritable. Estas dificultades están presentes en adultos con TDAH en ausencia de otros trastornos afectivos comórbidos, como la depresión y la ansiedad, y parecen normalizarse con el empleo de metilfenidato y, especialmente, de la atomoxetina (Reimherr et al., 2005). Un reciente estudio ha demostrado además que la atomoxetina no sólo mejora la labilidad emocional de los adultos con TDAH sino también la regulación emocional de 
niños y adolescentes con el trastorno (Wehmeier et al., 2008). Sin embargo, la eficacia del metilfenidato sobre el control emocional en niños y adolescentes no es tan clara. Por un lado, el estudio de Schachter et al. (Schachter, Pham, King, Langford y Moher, 2001) muestra que aunque el metilfenidato reduce la labilidad emocional de los niños con TDAH, esta reducción no resulta estadísticamente significativa. Por otro lado, varios estudios conductuales han observado dificultades en la regulación emocional en niños y adolescentes con TDAH a pesar de que gran parte de ellos estaban medicados con metilfenidato (Jensen y Rosen, 2004; Johnson \& Rosen, 2000).

Apenas se han explorado las bases neurobiológicas que subyacen a la pobre regulación emocional mostrada por los niños, adolescentes y adultos con TDAH (Passarotti, Sweeney y Pavuluri, 2010). No obstante, algunas de las principales regiones cerebrales implicadas en el control emocional, incluida la corteza dorsolateral y ventromedial (Lévesque et al., 2003; Ochsner, Bunge, Gross y Gabrieli, 2002), la corteza cingulada anterior (Albert, López-Martín y Carretié, 2010; Beauregard, Lévesque y Bourgouin, 2001) y la amígdala (Banks, Eddy, Angstadt, Nathan y Phan, 2007; Hare, Tottenham, Davidson, Glover y Casey, 2005), están alteradas en el TDAH, tanto desde el punto de vista anatómico (Perlov et al., 2008; Plessen et al., 2006) y neuroquímico (Volkow et al., 2007, 2009) como funcional (en este caso, por una disfunción en otros procesos psicológicos no vinculados con la regulación emocional: Bush et al., 2005; Scheres et al., 2007). Futuros estudios deberán explorar de manera directa si las dificultades para controlar las emociones de los niños y adultos con TDAH están vinculadas con una disfunción en estas regiones. Por el momento, los datos preliminares del grupo de Passarotti (2010) sugieren que una baja activación de la corteza prefrontal ventrolateral podría estar detrás de la pobre regulación emocional mostrada por muchos niños con TDAH.

\section{Implicaciones clínicas y direcciones futuras}

Esta revisión subraya la necesidad de evaluar y tratar las alteraciones emocionales y motivacionales del TDAH en la práctica clínica. Particularmente relevante es el estudio de Solanto et al. (2001) en el que se observó que la aversión a la demora explicaba mayor varianza de los síntomas del trastorno que la pobre inhibición de respuesta cuando ambas fueron medidas con test neuropsicológicos (Choice Delay task y Stopsignal task, respectivamente). Además, observaron que la aversión a la demora y el pobre control inhibitorio constituían dos alteraciones independientes que por sí solas no podían explicar todos los casos del trastorno pero que juntas lograban clasificar cerca del $90 \%$ de los niños con TDAH. Estos resultados han sido replicados posteriormente por otros estudios (Antrop et al., 2006; Sonuga-Barke et al., 2003; Thorell, 2007) y ponen de manifiesto la importancia de desarrollar para la práctica clínica test neuropsicológicos baremados que valoren las alteraciones motivacionales del TDAH. Según nuestro conocimiento, distintos grupos de investigación, entre los que se encuentran investigadores españoles, ya están desarrollando a nivel experimental pruebas neuropsicológicas para medir la aversión a la demora (Marco et al., 2009). La aplicación de estos test, además de los ya existentes para medir las alteraciones en las funciones ejecutivas (p.e., Continuous performance test), permitirá clasificar los distintos perfiles neuropsicológicos del trastorno. Además, la evaluación de marcadores neuropsicológicos, como el control inhibitorio o la aversión a la demora, podría jugar en el futuro un papel relevante en el diagnóstico del TDAH en sustitución de la actual evaluación basada en los síntomas conductuales (Sonuga-Barke, Sergeant, Nigg y Willcutt, 2008). Estos marcadores neuropsicológicos actuarían como endofenotipos o fenotipos intermedios entre los síntomas y las causas del trastorno, con las ventajas que este tipo de aproximación supondría (Castellanos y Tannock, 2002).

Los datos recogidos en esta revisión subrayan además la necesidad de llevar a cabo intervenciones educativas para incrementar la motivación de los alumnos con TDAH. Por un lado, la hipoactivación de la respuesta del sistema mesolímibico (particularmente, el estriado ventral) durante la anticipación de la recompensa explicaría las importantes dificultades que tienen muchos de los niños con TDAH para trabajar con refuerzos a largo plazo y para mantener la motivación en tareas monótonas y aburridas (tareas que no refuerzan pos sí mismas). Además, tanto los síntomas de inatención (Volkow et al., 2009) como los de hiperactividad e impulsividad (Carmona et al., 2009; Scheres et al., 2007; Ströhle et al., 2008) podrían estar también relacionados con las alteraciones neurobiológicas del sistema de recompensa. Por tanto, intervenciones educativas en las que se proporcione refuerzo externo, inmediato y frecuente podrían tener beneficios inmediatos en la reducción de la inatención, la hiperactividad y la impulsividad de los alumnos con TDAH, además de un aumento de su rendimiento académico. Por otro lado, las alteraciones en el sistema de recompensa y, en particular, del núcleo accumbens, recientemente observadas en el TDAH podrían explicar por qué los adolescentes y adultos con este trastorno son más susceptibles al abuso de sustancias que la población general. Determinadas drogas, incluida la anfetamina, la nicotina o el alcohol, tienen la capacidad, al igual que los reforzadores naturales, de aumentar la concentración de dopamina en el núcleo accumbens. En este sentido, el abuso de sustancias en el TDAH podría interpretarse como una forma de compensación o automedicación (Wilens et al., 2007), ya que, como indican algunos datos preliminares, los pacientes con este trastorno tienen una escasa concentración de dopamina en las regiones ventrales del estriado, incluido el núcleo accumbens y el caudado (Volkow et al., 2007, 2009). Esta hipótesis estaría en consonancia con los estudios clínicos que indican que el uso de metilfenidato durante la adolescencia reduce el riesgo de abuso de sustancias en los pacientes con TDAH (Faraone y Wilens, 2007; Wilens, Faraone, Biederman y Gunawardene, 2003). Por 
tanto, estos datos apoyarían el empleo continuado de metilfenidato durante la adolescencia y el comienzo de la vida adulta en pacientes con TDAH.

La Neurociencia afectiva del TDAH constituye una de las aproximaciones más prometedoras de este trastorno que complementará los importantes hallazgos que se han obtenidos durante la última década desde la Neurociencia cognitiva. Futuras investigaciones deberán explorar mediante distintas tareas experimentales y técnicas de registro, las bases neurobiológicas que subyacen a las alteraciones motivacionales y emocionales del TDAH, así como el efecto de los principales tratamientos farmacológicos sobre ellas. En este sentido, datos preliminares sugieren que el tratamiento con metilfenidato normaliza algunas de las alteraciones neurales asociadas con la motivación y la recompensa (Rubia et al., 2009) así como con el procesamiento emocional (Williams et al., 2008). La investigación sobre las alteraciones afectivas del TDAH y las bases neurobiológicas que las sustentan deberá tener especial cuidado en la selección de las muestra clínicas de pacientes con TDAH para no confundir las disfunciones afectivas propias de este trastorno con la de otros trastornos comórbidos. Finalmente, el estudio de la interacción entre los procesos afectivos y los procesos cognitivos, así como la comprensión de las bases neurobiológicas que las sustentan, representa una prometedora línea de investigación del TDAH (López-Martín et al., 2008; López-Martín, Albert, Fernández-Jaén, Tapia, Carretié, 2009; Passarotti et al., 2010).

\section{Conclusión}

La presente revisión pone de manifiesto que el TDAH es un trastorno complejo y heterogéneo en el que están implicados no sólo procesos cognitivos sino también afectivos. Muchas de las personas con TDAH muestran distintas alteraciones motivacionales, entre las que destaca la escasa habilidad para tolerar la demora de la recompensa. La aversión a la demora se manifiesta en una incapacidad para trabajar con recompensas a largo plazo, lo que provoca que el comportamiento de estas personas termine guiándose por recompensas inmediatas que muchas veces están alejadas de los objetivos deseados. La disfunción del circuito mesolímbico (corteza prefrontal ventromedial, núcleo accumbens y amígdala, fundamentalmente) parece estar detrás de las principales alteraciones motivacionales del TDAH, incluida la deficiente anticipación de la recompensa y la aversión a la demora. Asimismo, muchas de las personas con TDAH muestran alteraciones emocionales, entre las que destacan la dificultad para reconocer la información emocional y la incapacidad para regular sus propias emociones. Las investigaciones que han abordado el estudio de los mecanismos neurales implicados en el procesamiento emocional de las personas con TDAH sugieren la presencia de anomalías en etapas tempranas del procesamiento, tanto desde el punto de vista temporal como anatómico. Además, datos recientes apuntan a que un mal funcionamiento de la amígdala podría estar detrás de los pro- blemas en el procesamiento emocional observados en el TDAH (Brotman et al., 2010). Asimismo, datos preliminares sugieren la implicación de la corteza prefrontal ventrolateral en los problemas de regulación emocional del TDAH (Passarotti et al., 2009). Esta revisión subraya la necesidad de evaluar y tratar las alteraciones afectivas en la práctica clínica y continuar con la investigación sobre las mismas desde la Neurociencia afectiva.

\section{Referencias}

Albert, J., López-Martín, S. y Carretié, L. (2010). Emotional context modulates response inhibition: Neural and behavioral data. NeuroImage, 49, 914-921.

Albert, J., López-Martín, S., Fernández-Jaén, A. y Carretié, L. (2009). Trastorno por déficit de atención con o sin hiperactividad. JANO, 1729, 18-26.

Albert, J., López-Martin, S., Fernández-Jaen, A. y Carretie, L. (2008). Alteraciones emocionales en el trastorno por déficit de atención con hiperactividad: Datos existentes y cuestiones abiertas. Revista de Neurología, 47, 39-45.

Antrop, I. S. P., Verte, S. W. J. y Baeyens, D. R. H. (2006). ADHD and delay aversion: The influence of non-temporal stimulation on choice for delayed rewards. Journal of Child Psychology and Psychiatry, 47, 1152-1158.

Banks, S. J., Eddy, K. T., Angstadt, M., Nathan, P. J. y Phan, K. L. (2007). Amygdala frontal connectivity during emotion regulation. Social Cognitive and Affective Neuroscience, 2, 303-312.

Barkley, R. A. (1997). Behavioral inhibition, sustained attention, and executive functions: Constructing a unifying theory of ADHD. Psychological Bulletin, 121, 65-94.

Barry, R. J., Johnstone, S. J. y Clarke, A. R. (2003). A review of electrophysiology in attention-deficit/hyperactivity disorder: II. event-related potentials. Clinical Neurophysiology, 114, 184-198.

Beauregard, M., Lévesque, J. y Bourgouin, P. (2001). Neural correlates of conscious self-regulation of emotion. The Journal of Neuroscience, 21, 1-6.

Braaten, E. B. y Rosen, L. A. (2000). Self-regulation of affect in attention deficit-hyperactivity disorder (ADHD) and nonADHD boys: Differences in empathic responding. Journal of Consulting \& Clinical Psychology, 68, 313-321.

Brotman, M.A., Rich, B.A., Guyer, A.E., Lunsford, J.R., Horsey, S.E. y Reising, M.M. (2010). Amygdala activation during emotion processing of neutral faces in children with severe mood dysregulation versus ADHD or bipolar disorder. American Journal of Psychiatry, 167, 61-69.

Bush, G., Valera, E. M. y Seidman, L. J. (2005). Functional neuroimaging of attention-deficit/hyperactivity disorder: A review and suggested future directions. Biological Psychiatry, 57, 1273-1284.

Cadesky, E. B., Mota, V. L. y Schachar, R. J. (2000). Beyond words: How do problem children with ADHD and/or con 
duct problems process nonverbal information about affect? Journal of the American Academy of Child \& Adolescent Psychiatry, 39, 1160-1167.

Cardinal, R. N., Pennicott, D. R., Sugathapala, C. L., Robbins, T. W. y Everitt, B. J. (2001). Impulsive choice induced in rats by lesions of the nucleus accumbens core. Science, 292, 2499-2501.

Carmona, S., Proal, E., Hoekzema, E. A., Gispert, J. D., Picado, M., Moreno, I., et al. (2009). Ventro-striatal reductions underpin symptoms of hyperactivity and impulsivity in attention-Deficit/Hyperactivity disorder. Biological Psychiatry, 66, 972-977.

Carretié, L., Albert, J. y López-Martín, S. (2010). Papel de la corteza prefrontal ventromedial en la respuesta a eventos emocionalmente negativos. Revista de Neurología, 50, 245-252.

Carretié, L., Albert, J., López-Martín, S. y Tapia, M. (2009a). Negative brain: An integrative review on the neural processes activated by unpleasant stimuli. International Journal of Psychophysiology, 71, 57-63.

Carretié, L., Ríos, M., de la Gándara, B., Tapia, M., Albert, J., López-Martín, S., et al. (2009b). The striatum beyond reward: Caudate responds intensely to unpleasant pictures. Neuroscience, 164, 1615-1622.

Castellanos, F. X. y Tannock, R. (2002). Neuroscience of attention-deficit/hyperactivity disorder: The search for endophenotypes. Nature Reviews, 3, 617-628.

Clendenin, A. A., Businelle, M. S. y Kelley, M. L. (2005). Screening ADHD problems in the sports behavior checklist: Factor structure, convergent and divergent validity, and group differences. Journal of Attention Disorders, 8, 79-87.

Corbett, B. y Glidden, H. (2000). Processing affective stimuli in children with attention-deficit hyperactivity disorder. Child Neuropsychology, 6, 144-155.

Cortese, S., Angriman, M., Maffeis, C., Isnard, P., Konofal, E., Lecendreux, M. et al. (2008). Attention-deficit/hyperactivity disorder (ADHD) and obesity: A systematic review of the literature. Critical Reviews in Food Science and Nutrition, 48, 524-537.

Critchley, H. D., Daly, E. M., Bullmore, E. T., Williams, S. C. R., Van Amelsvoort, T., Robertson, D. M., et al. (2000). The functional neuroanatomy of social behaviour: Changes in cerebral blood flow when people with autistic disorder process facial expressions. Brain, 123, 2203-2212.

Da Fonseca, D., Seguier, V., Santos, A., Poinso, F. y Deruelle, C. (2009). Emotion understanding in children with ADHD. Child Psychiatry and Human Development, 40, 111-121.

Dalley, J. W., Fryer, T. D., Brichard, L., Robinson, E. S. J., Theobald, D. E. H., Laane, K., et al. (2007). Nucleus accumbens D2/3 receptors predict trait impulsivity and cocaine reinforcement. Science, 315, 1267-1270.

Dickstein, S., Bannon, K., Xaviercastellanos, F. y Milham, M. (2006). The neural correlates of attention deficit hyperactivity disorder: An ALE meta-analysis. Journal of Child
Psychology and Psychiatry, 47, 1051-1062.

Douglas, V. I. y Parry, P. A. (1983). Effects of reward on delayed reaction time task performance of hyperactive children. Journal of Abnormal Child Psychology, 11, 313-326.

Douglas, V. I. y Parry, P. A. (1994). Effects of reward and nonreward on frustration and attention in attention deficit disorder. Journal of Abnormal Child Psychology, 22, 281-302.

Faraone, S. V., Perlis, R. H., Doyle, A. E., Smoller, J. W., Goralnick, J. J., Holmgren, M. A., et al. (2005). Molecular genetics of attention-deficit/hyperactivity disorder. Biological Psychiatry, 57, 1313-1323.

Faraone, S. V. y Wilens, T. E. (2007). Effect of stimulant medications for attention-deficit/hyperactivity disorder on later substance use and the potential for stimulant misuse, abuse, and diversion. The Journal of Clinical Psychiatry, 68, 15-22.

Goldstein, R. Z. y Volkow, N. D. (2002). Drug addiction and its underlying neurobiological basis: Neuroimaging evidence for the involvement of the frontal cortex. American Journal of Psychiatry, 159, 1642.

Gross, J. J. (1998). The emerging field of emotion regulation: An integrative review. Review of General Psychology, 2, 271-299.

Hare, T. A., Tottenham, N., Davidson, M. C., Glover, G. H. y Casey, B. J. (2005). Contributions of amygdala and striatal activity in emotion regulation. Biological Psychiatry, 57, 624-632.

Herrmann, M. J., Schreppel, T., Biehl, S. C., Jacob, C., Heine, M., Boreatti-Hummer, A., et al. (2009). Emotional deficits in adult ADHD patients: An ERP study. Social Cognitive and Affective Neuroscience, 4, 340-345.

Holroyd, C. B., Baker, T. E., Kerns, K. A. y Müller, U. (2008). Electrophysiological evidence of atypical motivation and reward processing in children with attention-deficit hyperactivity disorder. Neuropsychologia, 46, 2234-2242.

Jensen, S. A. y Rosen, L. A. (2004). Emotional reactivity in children with attention-Deficit/Hyperactivity disorder. Journal of Attention Disorders, 8, 53-61.

Johnson, R. C. y Rosen, L. A. (2000). Sports behavior of ADHD children. Journal of Attention Disorders, 4, 150-160.

Kats-Gold, I., Besser, A. y Priel, B. (2007). The role of simple emotion recognition skills among school aged boys at risk of ADHD. Journal of Abnormal Child Psychology, 35, 363378.

Kitchens, S. A., Rosen, L. A. y Braaten, E. B. (1999). Differences in anger, aggression, depression, and anxiety between ADHD and non-ADHD children. Journal of Attention Disorders, 3, 77-83.

Lee, B., London, E. D., Poldrack, R. A., Farahi, J., Nacca, A., Monterosso, J. R., et al. (2009). Striatal dopamine D2/ D3 receptor availability is reduced in methamphetamine dependence and is linked to impulsivity. Journal of Neuroscience, 29, 14734. 
Lévesque, J., Eugène, F., Joanette, Y., Paquette, V., Mensour, B., Beaudoin, G., et al. (2003). Neural circuitry underlying voluntary suppression of sadness. Biological Psychiatry, 53, 502-510.

López Martín, S., Albert, J., Fernández-Jaén, A., Tapia, M. y Carretié, L. (2008). Selective attention to emotional visual stimuli in ADHD children: Preliminary electrophysiological data. International Journal of Psychophysiology, 69, 260-261.

López-Martín, S., Albert, J., Fernádez-Jaén, A., Tapia, M., Carretié, L. (2009). Interaction between automatic attention and emotion in Attention Deficit Hyperactivity Disorder: an event-related potential study. NeuroImage, 47, S181.

Luman, M., Oosterlaan, J., Hyde, C., van Meel, C. S. y Sergeant, J. A. (2007). Heart rate and reinforcement sensitivity in ADHD. Journal of Child Psychology and Psychiatry, 48, 890-898.

Luman, M., Oosterlaan, J. y Sergeant, J. A. (2005). The impact of reinforcement contingencies on $\mathrm{AD} / \mathrm{HD}$ : A review and theoretical appraisal. Clinical Psychology Review, 25, 183-213.

Luman, M., Tripp, G. y Scheres, A. (2010). Identifying the neurobiology of altered reinforcement sensitivity in ADHD: A review and research agenda. Neuroscience \& Biobehavioral Reviews, 34, 744-754.

Maedgen, J. W. y Carlson, C. L. (2000). Social functioning and emotional regulation in the attention deficit hyperactivity disorder subtypes. Journal of Clinical Child Psychology, 29, 30-42.

Marco, R., Miranda, A., Schlotz, W., Melia, A., Mulligan, A., Müller, U., et al. (2009). Delay and reward choice in ADHD: An experimental test of the role of delay aversion. Neuropsychology, 23, 367-380.

Marsh, P.J. y Williams, L.M. (2006). ADHD and schizophrenia phenomenology: Visual scanpaths to emotional faces as a potential psychophysiological marker? Neuroscience and Biobehavioral Reviews, 30,651-665.

Nigg, J. T. y Casey, B. J. (2005). An integrative theory of attention-deficit/ hyperactivity disorder based on the cognitive and affective neurosciences. Development and Psychopathology, 17, 785-806.

Norvilitis, J. M., Casey, R. J., Brooklier, K. M. y Bonello, P. J. (2000). Emotion appraisal in children with attentiondeficit/hyperactivity disorder and their parents. Journal of Attention Disorders, 4, 15-26.

Ochsner, K. N., Bunge, S. A., Gross, J. J. y Gabrieli, J. D. E. (2002). Rethinking feelings: An fMRI study of the cognitive regulation of emotion. Journal of Cognitive Neuroscience, 14, 1215-1229.

Passarotti, A.M, Sweeney, J.A. y Pavuluri, M.N. (2010). Differential engagement of cognitive and affective neural systems in pediatric bipolar disorder and attention deficit hyperactivity disorder. Journal of the International Neuropsychological Society, 16, 106-117.
Perlov, E., Philipsen, A., van Elst, L. T., Ebert, D., Henning, J., Maier, S., et al. (2008). Hippocampus and amygdala morphology in adults with attention-deficit hyperactivity disorder. Journal of Psychiatry \& Neuroscience, 33, 509-515.

Plessen, K. J., Bansal, R., Zhu, H., Whiteman, R., Amat, J., Quackenbush, G. A., et al. (2006). Hippocampus and amygdala morphology in attention-deficit/hyperactivity disorder. Archives of General Psychiatry, 63, 795-807.

Plichta, M. M., Vasic, N., Wolf, R. C., Lesch, K. P., Brummer, D., Jacob, C., et al. (2009). Neural hyporesponsiveness and hyperresponsiveness during immediate and delayed reward processing in adult attention-deficit/hyperactivity disorder. Biological Psychiatry, 65, 7-14.

Ramirez, C. A., Rosén, L. A., Deffenbacher, J. L., Hurst, H., Nicoletta, C., Rosencranz, T. y Smith, K. (1997). Anger and anger expression in adults with high ADHD symptoms. Journal of Attention Disorders, 2, 115-128.

Rapport, M. D., Tucker, S. B., DuPaul, G. J., Merlo, M. y Stoner, G. (1986). Hyperactivity and frustration: The influence of control over and size of rewards in delaying gratification. Journal of Abnormal Child Psychology, 14, 191-204.

Rapport, L. J., Friedman, S. R., Tzelepis, A. y Van Voorhis, A. (2002). Experienced emotion and affect recognition in adult attention-deficit hyperactivity disorder. Neuropsychology, 16, 102-110.

Reimherr, F. W., Marchant, B. K., Strong, R. E., Hedges, D. W., Adler, L. y Spencer, T. (2005). Emotional dysregulation in adult ADHD and response to atomoxetine. Biological Psychiatry, 58, 125-131.

Richards, T. L., Deffenbacher, J. L., Rosen, L. A., Barkley, R. A. y Rodricks, T. (2006). Driving anger and driving behavior in adults with ADHD. Journal of Attention Disorders, 10, 54-64.

Rolls, E.T. (2000). The Orbitofrontal Cortex and Reward. Cerebral Cortex, 10, 284-294.

Rubia, K., Halari, R., Cubillo, A., Abdul-Majeed Mohammad, A-M, Brammer, M. y Taylor, E. (2009). Methylphenidate normalises activation and functional connectivity deficits in attention and motivation networks in medication-naive children with ADHD during a rewarded continuous performance task. Neuropharmacology, 57, 640-652.

Sagvolden, T., Aase, H., Zeiner, P. y Berger, D. (1998). Altered reinforcement mechanisms in attention-deficit/hyperactivity disorder. Behavioural Brain Research, 94, 61-71.

Sagvolden, T., Johansen, E. B., Aase, H. y Russell, V. A. (2005). A dynamic developmental theory of attention-deficit/hyperactivity disorder (ADHD) predominantly hyperactive/impulsive and combined subtypes. The Behavioral and Brain Sciences, 28, 397-419.

Schachter, H. M., Pham, B., King, J., Langford, S. y Moher, D. (2001). How efficacious and safe is short-acting methylphenidate for the treatment of attention-deficit disorder in children and adolescents? A meta-analysis. Canadian 
Medical Association Journal, 165, 1475-1488.

Scheres, A., Milham, M. P., Knutson, B. y Castellanos, F. X. (2007). Ventral striatal hyporesponsiveness during reward anticipation in attention-deficit/hyperactivity disorder. Biological Psychiatry, 61, 720-724.

Schupp, H. T., Junghöfer, M., Weike, A. I. y Hamm, A. O. (2003). Emotional facilitation of sensory processing in the visual cortex. Psychological Science, 14, 7-13.

Seidman, L. J., Valera, E. M. y Makris, N. (2005). Structural brain imaging of attention-deficit/ hyperactivity disorder. Biological Psychiatry, 57, 1263-1272.

Shapiro, E. G., Hughes, S. J., August, G. J. y Bloomquist, M. L. (1993). Processing of emotional information in children with attention-deficit hyperactivity disorder. Developmental Neuropsychology, 9, 207-224.

Singh, S. D., Ellis, C. R., Winton, A. S., Singh, N. N., Leung, J. P. y Oswald, D. P. (1998). Recognition of facial expressions of emotion by children with attention-deficit hyperactivity disorder. Behavior Modification, 22, 128-142.

Sobanski, E., Banaschewski, T., Asherson, P., Buitelaar, J., Chen, W. y Franke, B. (en prensa). Emotional lability in children and adolescents with attention deficit/hyperactivity disorder (ADHD): Clinical correlates and familial prevalence. Journal of Child Psychology and Psychiatry.

Solanto, M. V., Abikoff, H., Sonuga-Barke, E., Schachar, R., Logan, G. D., Wigal, T., Hechtman, L., Hinshaw, S. y Turkel, E. (2001). The ecological validity of delay aversion and response inhibition as measures of impulsivity in AD/HD: A supplement to the NIMH multimodal treatment study of AD/HD. Journal of Abnormal Child Psychology, 29, 215-228.

Sonuga-Barke, E. J. S. (2002). Psychological heterogeneity in $\mathrm{AD} / \mathrm{HD}$ - a dual pathway model of behaviour and cognition. Behavioural Brain Research, 130, 29-36.

Sonuga-Barke, E. J. S. (2003). The dual pathway model of AD/ HD: An elaboration of neuro-developmental characteristics. Neuroscience and Biobehavioral Reviews, 27, 593604.

Sonuga-Barke, E., Taylor, E., Sembi, S. y Smith, J. (1992). Hyperactivity and delay aversion-I. the effect of delay on choice. Journal of Child Psychology and Psychiatry, 33, 387-398.

Sonuga-Barke, E. J., Dalen, L. y Remington, B. (2003). Do executive deficits and delay aversion make independent contributions to preschool attention-deficit/hyperactivity disorder symptoms? Journal of the American Academy of Child and Adolescent Psychiatry, 42, 13351342.

Sonuga-Barke, E. J. S., Sergeant, J. A., Nigg, J. y Willcutt, E. (2008). Executive dysfunction and delay aversion in attention deficit hyperactivity disorder: Nosologic and diagnostic implications. Child and Adolescent Psychiatric Clinics of North America, 17, 367-384.
Sprich, S., Biederman, J., Crawford, M. H., Mundy, E. y Faraone, S. V. (2000). Adoptive and biological families of children and adolescents with ADHD. Journal of the American Academy of Child \& Adolescent Psychiatry, 39, 1432-1437.

Ströhle, A., Stoy, M., Wrase, J., Schwarzer, S., Schlagenhauf, F. y Huss, M. (2008). Reward anticipation and outcomes in adult males with attention-deficit/hyperactivity disorder. NeuroImage, 39, 966-972.

Sung, V., Hiscock, H., Sciberras, E. y Efron, D. (2008). Sleep problems in children with attention-deficit/hyperactivity disorder: Prevalence and the effect on the child and family. Archives of Pediatrics \& Adolescent Medicine, 162, 336342.

Thorell, L. (2007). Do delay aversion and executive function deficits make distinct contributions to the functional impact of ADHD symptoms? A study of early academic skill deficits. Journal of Child Psychology and Psychiatry, 48, 10611070.

Tripp, G. y Alsop, B. (2001). Sensitivity to Reward Delay in Children with Attention Deficit Hyperactivity Disorder (ADHD). Journal of Child Psychology and Psychiatry, 42, 691-698

Tripp, G. y Wickens, J. (2008). Dopamine transfer deficit: A neurobiological theory of altered reinforcement mechanisms in ADHD. Journal of Child Psychology and Psychiatry, 49, 691-704.

Vaidya, C. J. y Stollstorff, M. (2008). Cognitive neuroscience of attention deficit hyperactivity disorder: Current status and working hypotheses. Developmental Disabilities Research Reviews, 14, 261-267.

Valera, E. M., Faraone, S. V., Murray, K. E. y Seidman, L. J. (2007). Meta-analysis of structural imaging findings in attention-deficit/hyperactivity disorder. Biological Psychiatry, 61, 1361-1369.

van West, D., Claes, S. y Deboutte, D. (2009). Differences in hypothalamic-pituitary-adrenal axis functioning among children with ADHD predominantly inattentive and combined types. European Child \& Adolescent Psychiatry, 18, 543-553.

Volkow, N. D., Wang, G. J., Kollins, S. H., Wigal, T. L., Newcorn, J. H. y Telang, F. (2009). Evaluating dopamine reward pathway in ADHD: Clinical implications. JAMA, 302, 1084-1091.

Volkow, N. D., Wang, G. J., Newcorn, J., Telang, F., Solanto, M. V., Fowler, J. S., Logan, J., Ma, Y., Schulz, K. y Pradhan, K. (2007). Depressed dopamine activity in caudate and preliminary evidence of limbic involvement in adults with attention-deficit/hyperactivity disorder. Archives of General Psychiatry, 64, 932-940.

Walcott, C. M. y Landau, S. (2004). The relation between disinhibition and emotion regulation in boys with attention deficit hyperactivity disorder. Journal of Clinical Child and Adolescent Psychology, 53, 772-782. 
Wehmeier, P. M., Schacht, A., Lehmann, M., Dittmann, R. W., Silva, S. G. y March, J. S. (2008). Emotional well-being in children and adolescents treated with atomoxetine for attention-deficit/hyperactivity disorder: Findings from a patient, parent and physician perspective using items from the pediatric adverse event rating scale (PAERS). Child and Adolescent Psychiatry and Mental Health, 2, 11.

Wender, P. H., Reimherr, F. W. y Wood, D. R. (1981). Attention deficit disorder ('minimal brain dysfunction') in adults. A replication study of diagnosis and drug treatment. Archives of General Psychiatry, 38, 449-456.

Wender, P. H., Reimherr, F. W., Wood, D. y Ward, M. (1985). A controlled study of methylphenidate in the treatment of attention deficit disorder, residual type, in adults. The American Journal of Psychiatry, 142, 547-552.

Wilens, T. E., Adamson, J., Sgambati, S., Whitley, J., Santry, A., Monuteaux, M. C. y Biederman, J. (2007). Do individuals with ADHD self-medicate with cigarettes and substances of abuse? results from a controlled family study of ADHD. American Journal on Addictions, 16, 14-23.

Wilens, T. E., Faraone, S. V., Biederman, J. y Gunawardene, S. (2003). Does stimulant therapy of attention-deficit/hyperactivity disorder beget later substance abuse? A meta-analytic review of the literature. Pediatrics, 111, 179-185.
Willcutt, E. G., Doyle, A. E., Nigg, J. T., Faraone, S. V. y Pennington, B. F. (2005). Validity of the executive function theory of attention-deficit/hyperactivity disorder: A metaanalytic review. Biological Psychiatry, 57, 1336-1346.

Williams, L. M., Palmer, D., Liddell, B. J., Song, L. y Gordon, E. (2006). The 'when'and 'where'of perceiving signals of threat versus non-threat. NeuroImage, 31, 458-467.

Williams, L. M., Hermens, D. F., Palmer, D., Kohn, M., Clarke, S., Keage, H., Clark, C. R. y Gordon, E. (2008). Misinterpreting emotional expressions in attention-Deficit/Hyperactivity disorder: Evidence for a neural marker and stimulant effects. Biological Psychiatry, 63,917-926.

Yuill, N. y Lyon, J. (2007). Selective difficulty in recognising facial expressions of emotion in boys with ADHD. general performance impairments or specific problems in social cognition? European Child \& Adolescent Psychiatry, 16, 398-404.

Zimmer, L. (2009). Positron emission tomography neuroimaging for a better understanding of the biology of ADHD. Neuropharmacology, 57,601-607.

Fecha de recepción: 23 de febrero de 2010 Fecha de aceptación: 1 de abril de 2010 